\title{
Coats Plus syndrome: a diagnostic and therapeutic challenge in pediatric gastrointestinal hemorrhage
}

\author{
Ferda Özbay Hoşnut ${ }^{1 \oplus}$, Gülseren Şahin ${ }^{1 \oplus}$, Meltem Akçaboy ${ }^{2 \oplus}$ \\ ${ }^{1}$ Division of Pediatric Gastroenterology, ${ }^{2}$ Department of Pediatrics, Dr. Sami Ulus Maternity and Children's Health and Diseases \\ Training and Research Hospital, Ankara, Turkey.
}

\begin{abstract}
Background. Cerebroretinal microangiopathy with calcifications and cysts formerly known as Coats plus syndrome is a rare multisystemic autosomal recessive disease that affects the eyes, brain, bone, and gastrointestinal system. Intestinal telangiectasia are components of vascular malformations characterized by gastrointestinal system bleedings. Recurrent gastrointestinal system bleedings have been reported as being due to hepatic failure or vascular malformations of the gastrointestinal system tract.

Case. Here we report a patient who presented with recurrent gastrointestinal system bleeding episodes, bilateral exudative retinopathy, intracranial calcification and was diagnosed with Coats plus syndrome. Recurrent gastrointestinal system bleeding was controlled by monthly octreotide treatment.

Conclusions. Coats plus syndrome presenting with vascular malformations should always be kept in mind in a patient with recurrent gastrointestinal bleeding and accompanying systemic physical findings. Octreotide treatment is an important option for patients with life threatening gastrointestinal system bleeding. Long term use of octreotide treatment can be used successfully in selected pediatric cases.
\end{abstract}

Key words: gastrointestinal bleeding, Coats plus syndrome, octreotide.

Gastrointestinal (GI) hemorrhage is one of the most serious complaints observed in pediatric practice. There are many causes of GI bleeding ranging from those requiring urgent treatment to those that resolve without any therapy. Determining why this is occurring is the art of any medical diagnostic approach and therapeutic options are not always available. Vascular malformations are a rare cause of GI bleeding in children and they are generally difficult to identify. ${ }^{1,2}$ We present an adolescent boy with recurrent severe GI bleeding who was diagnosed with Coats plus syndrome (CpS), due to a mutation in the conserved telomere maintenance component 1 (CTC1) gene, in order to relate the importance of a multisystem evaluation of pediatric patients with GI bleeding and also to discuss the treatment options.

$\triangle$ Meltem Akçaboy

meltemileri@yahoo.com

Received 2nd March 2021, revised 11th April 2021, accepted 16th April 2021.

\section{Case Report}

A 15-year-old male patient was admitted to our clinic with a history of fatigue and recurrent episodes of syncope during a 1-week period. He was born from a consanguineous family (first degree cousins) at full-term gestation after an uncomplicated pregnancy with a birth weight of 1550 grams with severe intrauterine growth retardation. His prenatal and natal history was uneventful except for intrauterine growth retardation. His past medical history included delayed psychomotor development and impaired vision. His developmental milestones were reported to be delayed. An ophthalmologic evaluation at 9 years of age revealed retinal vascular anomalies and capillary telangiectasias requiring laser photocoagulation. His history revealed a syncope 2 months before admission, and his evaluation in another center showed severe anemia. Red blood cell transfusion and oral iron supplementation was the prominent treatment. Physical examination revealed his 
growth parameters were within normal limits. He was pale. He had a white forelock in his hair. Lateral gaze palsy was noted in the right eye while medial gaze palsy was prominent in the left eye. Contracture was noted on the left hand. Loss of muscle power was evident in both the upper and lower left extremities. Deep tendon reflexes were increased on the left side. Left-sided dysmetria was noted on cerebellar examination. Choreoathetoid movements were prominent when he lifted his left arm. The remainder of the physical examination was normal.

Laboratory data revealed severe iron deficiency anemia with a hemoglobin level of $5,9 \mathrm{~g} / \mathrm{dL}$. In the laboratory evaluation, all the results including liver function tests were within normal ranges except for anemia. Gastroduodenoscopy with duodenal biopsy showed a duodenal ulcer with Helicobacter pylori. The patient was transfused with red blood cells and received eradication therapy for Helicobacter pylori. He was discharged for outpatient follow-up.

One month later the patient presented melena with a hemoglobin level of 7,1 g/dl. Repeated gastroduodenoscopy showed hyperemic lesions on the bulbus and antrum. No active bleeding source was demonstrated. The colonoscopy and scintigraphy screening for bleeding was normal. The patient was treated conservatively with red blood cell transfusion, proton pump inhibitors and oral propranolol, and then discharged and sent home.

The patient was admitted with a second episode of melena with severe anemia with a hemoglobin level of 7,6 g/dl. Double-balloon enteroscopy was performed, visualizing 200 $\mathrm{cm}$ after the pylorus. Most of the mucosa was covered with millimetric ulcers and severe vascularity was noted (Fig. 1). A biopsy revealed edema. Intravenous infusion of octreotide treatment was initiated. The bleeding stopped within days.

The patient was further evaluated for differential diagnosis, due to the accompanying neurological findings. Magnetic resonance imaging (MRI) and computerized tomography of the brain demonstrated amorphous dystrophic calcifications in both temporal lobes and left parietal lobes as well as widespread ischemia in both the thalamus and retrotrigonal periventricular area (Fig. 2). These findings, in conjunction with a physical examination evaluation for neurological, GI, eye and hair outcomes, were suggestive of Coats plus disease. Next generation sequencing identified a homozygous mutation c.2714G>A (p. Arg905Q) (p. Arg905Gln) in the CTC1 gene confirming $\mathrm{CpS}$. The patient was discharged with monthly intramuscular octreotide treatment. The patient has since been followed for 3 years without any GI bleeding and anemia. Informed consent was obtained from his parents for publication and photographs.

\section{Discussion}

We present a case of CpS admitted to our hospital for recurrent severe GI bleeding. The patient's accompanying neurological, ophthalmological, and hair findings along with intestinal lesions were the prominent components for the differential diagnosis. Further genetic evaluation is essential to confirm the diagnosis. The treatment of patients is planned individually according to the bleeding recurrence and accompanying risk factors. The patient benefited from monthly octreotide treatment and showed marked progress.

Coats plus syndrome is an autosomal recessive disease characterized by abnormalities in the eyes, brain, bones, GI system and other parts of the body. In addition, low birth weight, anemia, osteopenia, GI bleeding, portal hypertension, gray hair and nail dystrophies are frequently observed. ${ }^{3}$ Recurrent, severe GIS bleeding is increasingly identified in $\mathrm{CpS}$. In addition, cerebral microangiopathy, leukodystrophy, seizures, movement disorders, skeletal disorders and postnatal growth retardation were all reported. ${ }^{4}$ Our patient had all of the reported components of $\mathrm{CpS}$ with retinal telangiectasias, 


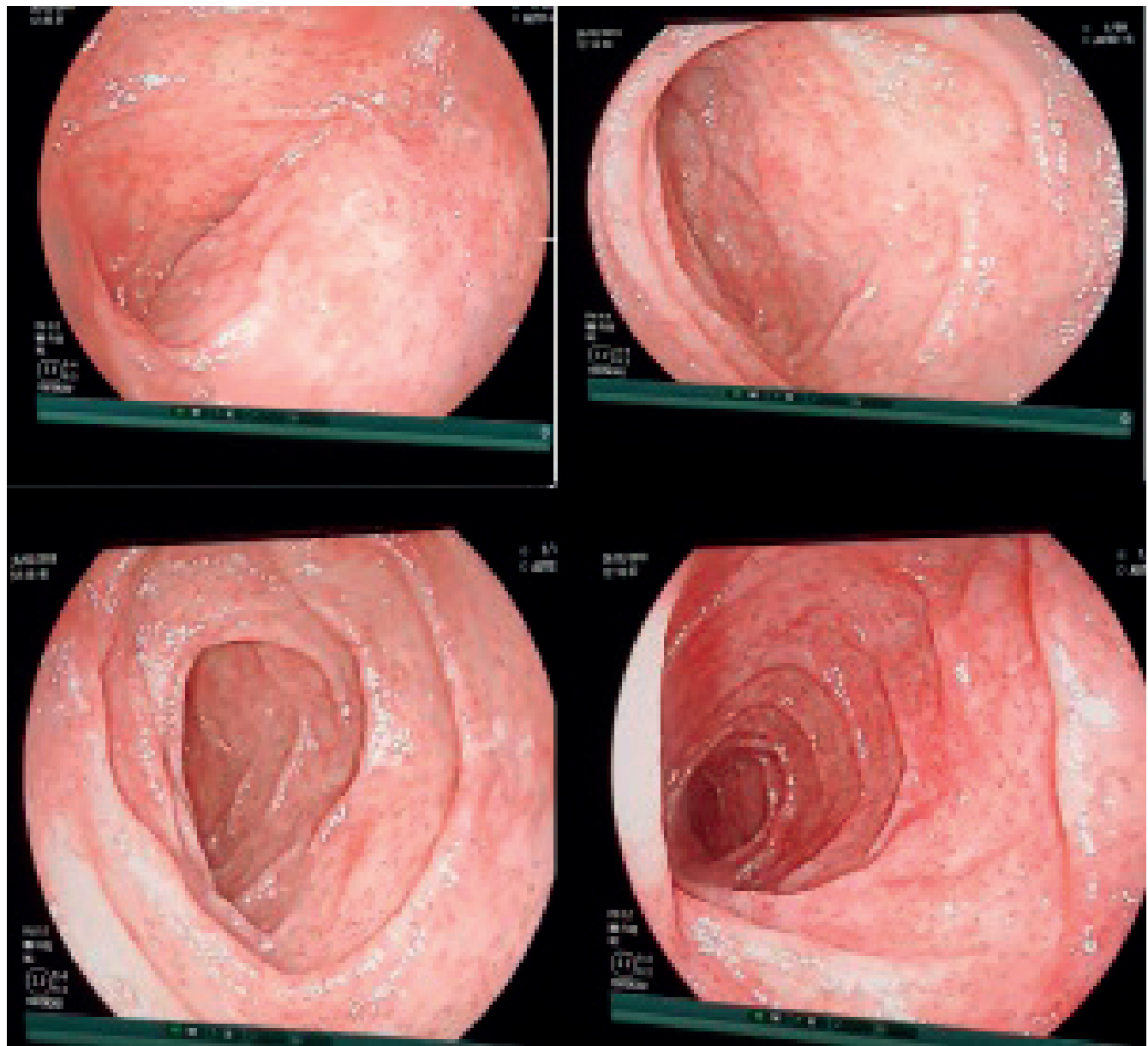

Fig. 1. Double-balloon enteroscopy showing millimetric ulcers and severe vascularity on intestines.

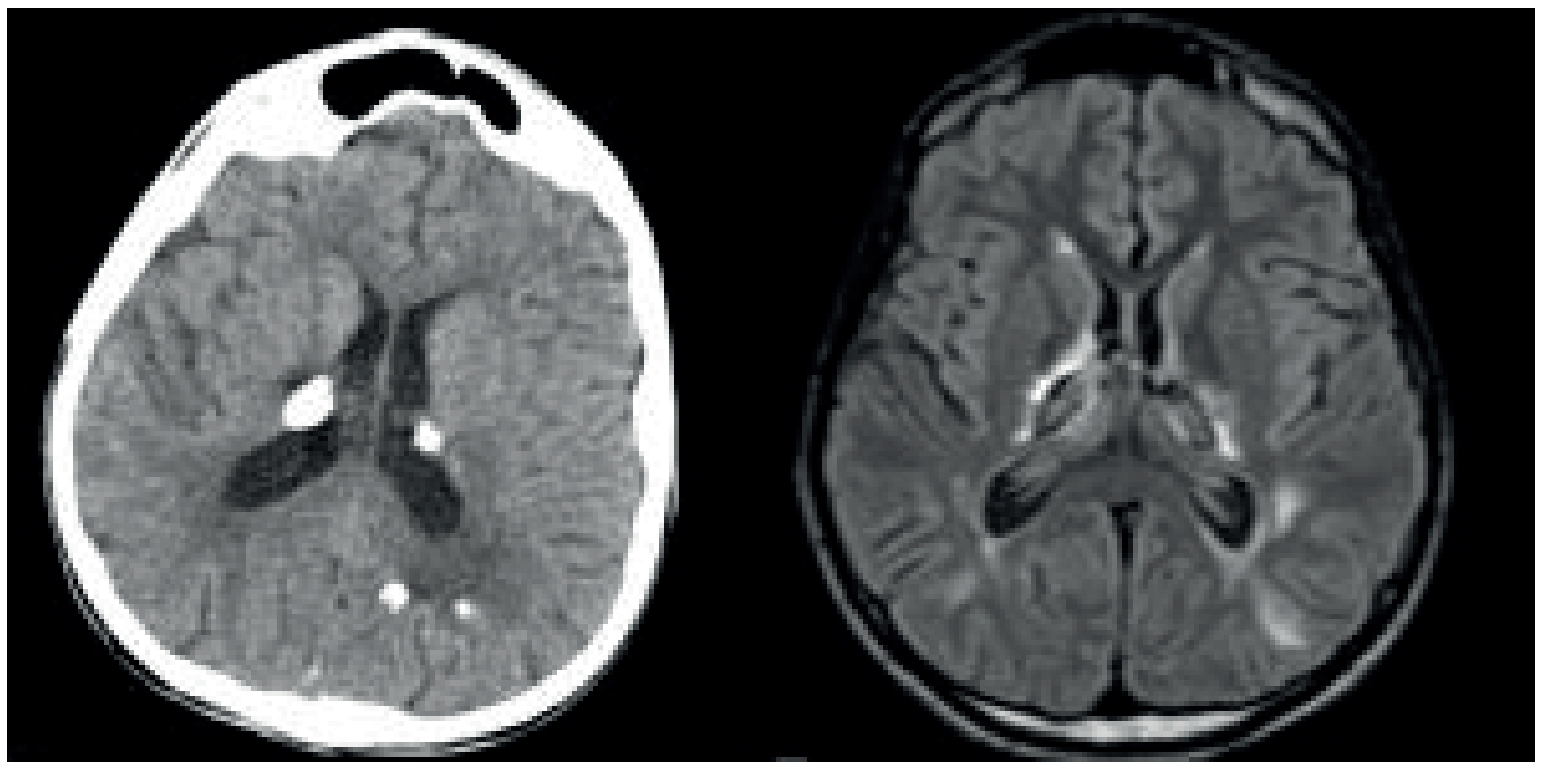

Fig. 2. Cranial computerized tomography scan showing variable and asymmetrically distributed calcifications (Left); Magnetic resonance imaging showed ischemia, gliotic regions and calcifications in FLAIR series (Right). 
intracranial calcification, psychomotor retardation, a history of being small for his gestational age, osteopenia, recurrent GI bleeding, liver parenchymal disease and portal hypertension. The diagnosis was a challenge as combining the components was not easy on the first admission. The genetic mutation in the CTC1 gene was first identified in 2012 (3); thus, further evaluation is necessary to confirm the diagnosis. We identified a previously reported mutation in the CTC1 gene.

GI bleeding in CpS is an important and lifethreatening component of the disease. The cause of bleeding is usually reported to be telangiectasias in the stomach and small intestine. Nevertheless, esophageal variceal bleeding secondary to the development of portal hypertension due to vascular telangiectasia in the liver is not rare. ${ }^{2}$ The main pathophysiology for the vascular lesions is obliterative microangiopathy, characterized by thick, sclerotic and calcified vascular walls detected in the eye and brain biopsies of patients with CpS. ${ }^{5}$ Our patient had non-cirrhotic portal hypertension. Gastroduodenoscopy and colonoscopy were inadequate for the diagnosis; so double-balloon enteroscopy was needed to show the vascular lesions.

Treatment options in vascular bleeding of the GI system in $\mathrm{CpS}$ are generally a challenge since the lesions are often multiplexed. In such cases, endoscopic and surgical treatments may be insufficient. ${ }^{6}$ The availability of octreotide in GI system malformations has been demonstrated in many studies. Subcutaneous octreotide therapy has been reported in patients with chronic GI system bleeding due to multiple vascular malformations. ${ }^{7}$ Continued monthly subcutaneous octreotide treatment for GI bleeding for intestinal vascular malformations has been reported for up to 15 years. ${ }^{8}$ Octreotide treatment has also been an option to control GI bleeding due to angiodysplasia in certain patients. $^{2}$ Therefore, we started octreotide treatment for our patient. During the three years of follow-up, our patient's bleeding did not recur and there was no anemia.

It is important to determine the etiology in determining effective treatment approaches in childhood GIS bleeding. However, indicating the exact cause is not always possible or can sometimes be delayed. In this process, the close monitoring and supportive treatment of the patient gain importance. In any GI system when bleeding cannot be controlled in children and adolescents, long term octreotide treatment is also an effective and sustainable treatment option in children. CpS should be considered as a rare cause of GI system bleeding in patients with multisystem involvement. Considering a multisystemic disease that also affects the GI system should be taken into account with any accompanying findings as we have reported here with our patient.

\section{Author contribution}

The authors confirm contribution to the paper as follows: study conception and design: EB, EG; data collection: EB, SB; analysis and interpretation of results: $\mathrm{EB}, \mathrm{TK}, \mathrm{MR}$; draft manuscript preparation: TK, ET. All authors reviewed the results and approved the final version of the manuscript.

\section{Conflict of interest}

The authors declare that there is no conflict of interest.

\section{REFERENCES}

1. Briggs TA, Hubbard M, Hawkins C, et al. Treatment of gastrointestinal bleeding in a probable case of cerebroretinal microangiopathy with calcifications and cysts. Mol Syndromol 2011; 1: 159-162. https:// doi.org/10.1159/000321559

2. Puri K, Caldwell RL, Molleston JP. Role of octreotide in pediatric gastrointestinal bleeding secondary to angiodysplasia in children with right heart failure. J Pediatr Gastroenterol Nutr 2018; 66: e41-e44. https:// doi.org/10.1097/MPG.0000000000001659 
3. Anderson BH, Kasher PR, Mayer J, et al. Mutations in CTC1, encoding conserved telomere maintenance component 1, cause Coats plus. Nat Genet 2012; 44: 338-342. https://doi.org/10.1038/ng.1084

4. Crow YJ, McMenamin J, Haenggeli CA, et al. Coats' plus: a progressive familial syndrome of bilateral Coats' disease, characteristic cerebral calcification, leukoencephalopathy, slow pre- and post-natal linear growth and defects of bone marrow and integument. Neuropediatrics 2004; 35: 10-19. https:// doi.org/10.1055/s-2003-43552

5. Briggs TA, Abdel-Salam GM, Balicki M, et al. Cerebroretinal microangiopathy with calcifications and cysts (CRMCC). Am J Med Genet Part A 2008; 146: 182-190. https://doi.org/10.1002/ajmg.a.32080
6. Polese L, Angriman I, Pagano D, et al. Laser therapy and surgical treatment in transfusion-dependent patients with upper-gastrointestinal vascular ectasia. Lasers Med Sci 2006; 21: 140-146. https://doi. org/10.1007/s10103-006-0389-7

7. Nardone G, Rocco A, Balzano T, Budillon G. The efficacy of octreotide therapy in chronic bleeding due to vascular abnormalities of the gastrointestinal tract. Alimen Pharmacol Therap 1999; 13: 1429-1436. https://doi.org/10.1046/j.1365-2036.1999.00647.x

8. Classen CF, Haffner D, Hauenstein C, Wolf R, Kyank U. Novel treatment (new drug/intervention; established drug/procedure in new situation): long-time octreotide in an adolescent with severe hemorrhagic gastrointestinal vascular malformation. BMJ Case Reports 2011; 2011: 15-20. https://doi. org/10.1136/bcr.05.2011.4291 